\title{
A New Integral Second-Order Terminal Sliding Mode Control with Time Delay Estimation for an Exoskeleton Robot with Dynamics Uncertainties
}

\author{
Brahim Brahmi ${ }^{1}$, Maarouf Saad ${ }^{1}$, Cristobal Ochoa-Luna ${ }^{1}$, Stefano Di Gennero ${ }^{2}$, Mohammed H. Rahman ${ }^{3}$ \\ ${ }^{1}$ École de technologie Supérieure \\ Montréal, Canada \\ brahim.brahmi.1@ens.etsmtl.ca; Maarouf.Saad@etsmtl.ca \\ ${ }^{2}$ Department of Information Engineering, Computer Science and Mathematics, University of L'Aquila \\ Aquila, Italy \\ Stefano.digennaro@univq.it \\ ${ }^{3}$ University of Wisconsin-Milwaukee \\ Milwaukee, Wisconsin, USA
}

\begin{abstract}
This paper presents a new Integral Second-Order Terminal Sliding Mode Control incorporating Time Delay Estimation applied to passive rehabilitation protocols of an exoskeleton robot with dynamics uncertainties and unknown bounded disturbances. The use of second-order sliding mode is due to its attractive characteristics of accuracy, attenuation of chattering and fast convergence. However, its problem is that the unknown dynamics of the exoskeleton robot and external disturbances caused by its different wearers can be amplified by the second derivative of the sliding surface, which leads to instability of the exoskeleton system. Using Time Delay Estimation will estimate the uncertain dynamics while overcoming the main limitation of second-order sliding mode. The stability analysis is formulated and proved based on Lyapunov function. Experimental results with a healthy subject confirm the effectiveness of the proposed control.
\end{abstract}

Keywords: Rehabilitation Robots, Second Order Sliding Mode; Time Delay Estimation, Passive Assistive Motion.

\section{Introduction}

Recently, the use of physiotherapy rehabilitation robots has shown great potential for improving the patient's disability and independence of function [1]. Control of these kind of robots presents additional complexity over the control of conventional robotic manipulators due to their complex mechanical structure designed for human use, the types of desired tasks, and the sensibility of the interaction with a large diversity of human wearers [1]. As a result, these conditions, make the robot system vulnerable to dynamics uncertainties and external disturbances.

Sliding mode control (SMC) is one of the most popular control strategies that is widely applied on robotics systems thanks to its attractive characteristics of robustness to the dynamics nonlinear-uncertainties and external disturbances [2]. However, conventional SMC suffers from two major shortcomings. The first one is that SMC ensures an asymptotic convergence to the equilibrium without finite-time convergence. Many control techniques have been developed to overcome this problem such terminal sliding mode control (TSMC) [3]. This later utilizes a nonlinear switching surface to guarantee the finite time convergence by including a fractional order, which allows to the states trajectories to converge to equilibrium faster. In literature, the accuracy performance of TSMC is improved by proposing a new approach for instance, fast TSMC [4] and non-singular TSMC [5]. A second major problem is that SMC is fundamentally based on a larger highgain switching controller which pushes the system state to converge to the equilibrium. Nevertheless, the high-activity switching gain causes an undesirable "chattering" dilemma which can damage the actuators of the robot system [6].

Recently, many conventional approaches were developed to avoid the undesirable chattering problem; e.g. by exchanging the discontinuous function by a continuous function (as a saturation function). Second Order Sliding Mode Controller (SOSMC) [7] is considered as one of the efficient approaches dedicated to eliminate chattering problem and provide a high performance's precision. Additionally, various approaches have been developed to improve the 
performance of SOSMC such Twisting control and Super-Twisting control [8]. The main idea of SOSMC is to allow a sliding surface and its consecutive derivative to go to zero and to maintain the discontinuous control under an integral function, which can eliminate the undesirable chattering. Nevertheless, the second-time derivative of the sliding surface might produce instability of the system, a risk that the nonlinear uncertainties and external disturbances amplify. Recently, Second Order Terminal Sliding mode Control (SOTSMC) was introduced to provide a great control performance to deal with a chattering phenomenon and provide a finite time convergence $[9,10]$. So, to the best of our knowledge, no SOTSMC with integral action has been proposed before to solve the mentioned problems.

Motivated to deal with the mentioned problem, and based on our previous work $[11,12]$, we proposed a new integral Second Order Terminal Sliding mode controller (ISOTSMC) combined with Time Delay Estimation (TDE) [12] to provide a good approximation of the uncertainties and the bounded external disturbances of an exoskeleton robot. TDE uses timedelayed knowledge about the previous system state and its control input to provide an accurate estimation of unknown dynamics. The incorporation of integral control relies on its attractive characteristics, where it has delivered good performance with conventional SMC [13]. The control scheme aims to keep the high precision of the SOSMC, eliminate the chattering problem, and provide a finite-time convergence to equilibrium.

The remainder of the paper is organized as follows. The dynamics of the robot is presented in the next section. The control scheme is described in section 3. Experimental results and some comparisons are given in section 4. Finally, the conclusion is presented in section 5 .

\section{Characterization of System Rehabilitation}

\subsection{Exoskeleton Robot Development}

The developed exoskeleton robot ETS-MARSE (École de technologie supérieure - Motion Assistive Roboticexoskeleton for Superior Extremity) is a redundant robot consisted of 7-degrees of freedom (DOFs), as shown in Fig. 1. It was created to provide assistive physiotherapy motion to the injured upper limb. The idea of the designed exoskeleton is basically extracted from the anatomy of the upper limb of the human, to be ergonomic for their wearer along the physiotherapy session. The shoulder part consists of three joints, the elbow part comprises by one joint and the wrist part consists of three joints. Each part responsible for performing a variety of upper limb motions. All special characteristics of the ETS-MARSE, the modified Denavit-Hartenberg (DH) parameters, and comparison with similar existing exoskeleton robots are summarized in [14].

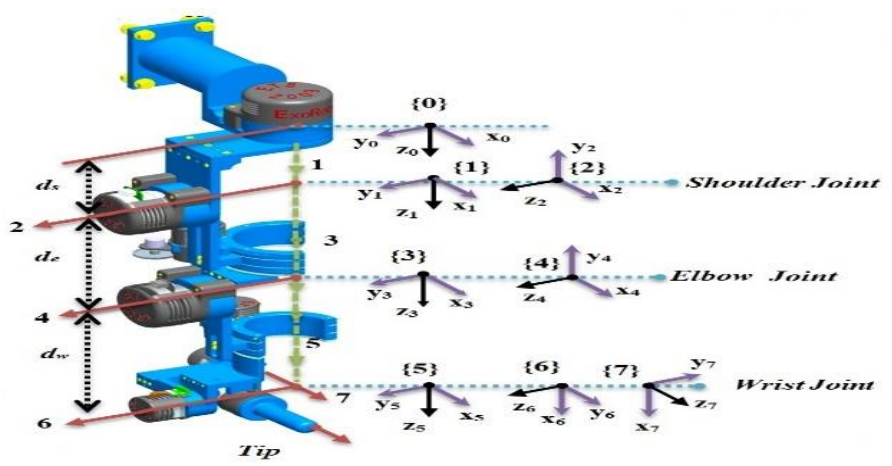

Fig. 1: Reference frames of ETS-MARSE.

\subsection{Dynamics of ETS-MARSE Robot}

The dynamics of ETS-MARSE is expressed as follows:

$$
M(\theta) \ddot{\theta}+C(\theta, \dot{\theta}) \dot{\theta}+G(\theta)+f_{d i s}=\tau
$$

where $\theta, \dot{\theta}$, and $\ddot{\theta} \in \mathbb{R}^{7}$ are respectively the joints position, velocity, and acceleration vectors, $M(\theta) \in \mathbb{R}^{7 \times 7}, C(\theta, \dot{\theta}) \dot{\theta} \in$ $\mathbb{R}^{7}$, and $G(\theta) \in \mathbb{R}^{7}$ are respectively the symmetric positive-definite inertia matrix, the Coriolis and centrifugal vector, and 
the gravitational vector including the user's arm and the exoskeleton arm. $\tau \in \mathbb{R}^{7}$ is the torque vector, $f_{\text {dis }} \in \mathbb{R}^{7}$ is the external disturbances vector. Without loss of generality, the dynamic model (1) can be rewritten as follows:

$$
\left\{\begin{aligned}
M(\theta) & =M_{0}(\theta)+\Delta M(\theta) \\
C(\theta, \dot{\theta}) & =C_{0}(\theta, \dot{\theta})+\Delta C(\theta, \dot{\theta}) \\
G(\theta) & =G_{0}(\theta)+\Delta G(\theta)
\end{aligned}\right.
$$

where $M_{0}(\theta), C_{0}(\theta, \dot{\theta})$, and $G_{0}(\theta)$ are respectively the known inertia matrix, the Coriolis/centrifugal matrix, and the gravity vector. $\Delta M(\theta), \Delta C(\theta, \dot{\theta})$, and $\Delta G(\theta)$ are the uncertain parts. Let us introduce a new variable such that: $z_{1}=\theta$ and $Z_{2}=\dot{\theta}$; hence, the dynamic model expressed in Eq. 1 can be rewritten as follows:

$$
\left\{\begin{array}{c}
\dot{z}_{1}=z_{2} \\
\dot{z}_{2}=U(t)+f(t)+H(t)
\end{array}\right.
$$

where, $U(t)=U\left(z_{1}\right) ; H(t)=H\left(z_{1}, z_{2}, \dot{z}_{2}\right)$ and $f(t)=f\left(z_{1}, z_{2}\right)$. This notation is used to facilitate the handling of the control methodology with: $U(\mathrm{t})=M_{0}^{-1}(\theta) \tau(t) ; H(\mathrm{t})=M_{0}^{-1}(\theta)\left(-f_{\text {dis }}-\Delta M(\theta) \ddot{\theta}-\Delta C(\theta, \dot{\theta}) \dot{\theta}-\Delta G(\theta)\right)$, and $f(\mathrm{t})=$ $M_{0}^{-1}(\theta)\left(-C_{0}(\theta, \dot{\theta}) \dot{\theta}-G_{0}(\theta)\right)$.

\subsection{Problem Statement}

The developed approach aims to set up a new integral Second-Order Terminal Sliding mode control (ISOTSMC) to improve the performance of conventional second-order SMC and to ensure the finite-time convergence of the sliding surface. Since the dynamic parameters of the robot are unknown, the integration of TDE to estimate them ensures a desirable performance. The control strategy is developed to be able to complete the passive rehabilitation movement by obtaining a control input that forces the measured trajectory to track the desired trajectory even if the robot operates with uncertain dynamics and unforeseen external disturbances.

Property 1: The known part of inertia matrix $M_{0}(\theta)$ is symmetric and positive definite for all $\theta \in \mathbb{R}^{n}$ [2].

Assumption 1: The function $H(t)$ is globally Lipschitz function.

Assumption 2: The desired trajectory is bounded.

Assumption 3: The external disturbance $f_{\text {dis }}$ is supposed to be continuous, has finite energy, and satisfies $\left\|f_{\text {dis }}\right\| \leq \varepsilon$, with an unknown positive disturbance boundary $\varepsilon$.

\section{Control Design}

The first step in the control development is to define the surface $S$ in terms of position error. Then, select the integral terminal type of the sliding surface, where this later must be stable and guarantee the finite-time convergence. Let us chose the integral terminal surface as follows:

$$
S=\lambda_{1} e+\lambda_{2} \int_{0}^{t}|e|^{\beta} \operatorname{sign}(e) d y
$$

where $e=z_{1}-z^{d}$ is the position error and $z_{1}, z^{d} \in \mathbb{R}^{7}$ is the measured and desired trajectory respectively, where $\lambda_{1}=\operatorname{diag}\left(\lambda_{1 i i}\right)>0, \lambda_{2}=\operatorname{diag}\left(\lambda_{2 i i}\right)>0$ where $i=1, \ldots, 7$; and $\frac{1}{2}<\beta<1$. Taking the first-time derivative of $S$, we find:

$$
\dot{S}=\lambda_{1} \dot{e}+\lambda_{2}|e|^{\beta} \operatorname{sign}(e)
$$

Theorem 1: Considering the exoskeleton robot system (Eq. 3) that satisfies the mentioned properties and assumptions, the selected surface (Eq. 4) is stable and finite-time independently of the initial state. 
Proof:_Let us consider the following Lyapunov function:

$$
V_{e}=\frac{1}{2} \sum_{i=1}^{7} e_{i}^{2}
$$

where $V_{e}\left(e_{0}\right)$ is the initial value of the selected Lyapunov function. The time derivative of Eq. 6 can be obtained by:

$$
\dot{V}_{e}=\sum_{i=1}^{7} e_{i} \dot{e}_{i}
$$

Let us assume that $\dot{S}=0$ is provided, from Eq. 5 we can obtain the following expression using scalar form as follows:

$$
\dot{e}_{i}=-\frac{\lambda_{2 i}}{\lambda_{1 i}}\left|e_{i}\right|^{\beta} \operatorname{sign}\left(e_{i}\right) ; \text { where } i=1, \ldots, 7
$$

Substituting Eq. 8 into Eq.7 we have:

$$
\begin{aligned}
\dot{V}_{e} & =-\sum_{i=1}^{7} \frac{\lambda_{2 i}}{\lambda_{1 i}}\left|e_{i}\right|^{\beta} e_{i} \operatorname{sign}\left(e_{i}\right) \\
& \leq-\sum_{i=1}^{7} \frac{\lambda_{2 i}}{\lambda_{1 i}}\left(e_{i}{ }^{2}\right)^{\frac{\beta+1}{2}} \\
& =-\sum_{i=1}^{7} \frac{\frac{\beta}{2+1}^{\frac{\beta+1}{2}} \lambda_{2 i}}{\lambda_{1 i}}\left(V_{e}\right)^{\frac{\beta+1}{2}}
\end{aligned}
$$

where $\left|e_{i}\right|=e_{i} \operatorname{sign}\left(e_{i}\right)$. Therefore, $\dot{V}_{e} \leq 0$ is verified. We can rewrite Eq. 9 as follows:

$$
\dot{V}_{e}+\sum_{i=1}^{7} \vartheta V_{e}^{\mu} \leq 0
$$

where $\vartheta=\frac{2^{\frac{\beta+1}{2}} \lambda_{2 i i}}{\lambda_{1 i i}}$ and $\mu=\frac{\beta+1}{2}$, taking into consideration that $\frac{1}{2}<\beta<1$ and $\frac{3}{4}<\mu<1$. So, according to [15], the convergence of the finite time $t_{s}$ can be given by:

$$
t_{s}=\frac{V_{e}^{1-\mu}\left(e_{0}\right)}{\vartheta(1-\mu)}
$$

where $V_{e}\left(e_{0}\right)$ is the Lyapunov function's initial value. The proof is complete.

Remark 1: It is obvious from Eq. 11 that the initial value of the Lyapunov function $V_{e}\left(e_{0}\right)$ and the ratio $\lambda_{2 i} / \lambda_{1 i}$ manage the finite time convergence $t_{s}$ of the selected sliding surface. A large value of $\lambda_{2 i} / \lambda_{1 i}$ can ensure a short convergence time. Likewise, too large gain ratio may produce an overshoot influence. Therefore, the trade-off between fast convergence and control performance is required to choose $\lambda_{1 i}$ and $\lambda_{2 i}$.

While the selected surface is chosen, the combination of ISOTSMC with TDE can be easily making up now. Let us take the second-time derivative of Eq. 5 as:

$$
\ddot{S}=\lambda_{1} \ddot{e}+\sum_{i=1}^{7} \beta \lambda_{2 i}|e|^{\beta-1} \dot{e}_{i}
$$

Substituting Eq. 8 into Eq. 12, we obtain:

$$
\ddot{S}=\lambda_{1}\left(U(t)+f(t)+H(t)-\ddot{Z}^{d}\right)-\sum_{i=1}^{7} \frac{\beta \lambda_{2 i}{ }^{2}}{\lambda_{1 i}}|e|^{2 \beta-1} \operatorname{sign}\left(e_{i}\right)
$$

To solve Eq. 13, the integral terminal super-twisting controller is given as follows: 


$$
U(t)=-k_{1} \lambda_{1}|\dot{S}|^{\frac{1}{2}} \operatorname{sign}(S)+\ddot{Z}^{d}-f(t)-H(t)+\sum_{i=1}^{7} \frac{\beta \lambda_{2 i}{ }^{2}}{\lambda_{1 i}{ }^{2}}|e|^{2 \beta-1} \operatorname{sign}\left(e_{i}\right)-\int_{0}^{t} k_{2} \lambda_{1} \operatorname{sign}(S)
$$

with $=M_{0} U(t), k_{1}=\operatorname{diag}\left(k_{1 i i}\right)>0$, and $k_{2}=\operatorname{diag}\left(k_{2 i i}\right)>0$, where $i=1, \ldots, 7$.

Practically, as established, all dynamic parameters of the exoskeleton robot are not easily obtained due to the uncertainties and their variation during the robot's tasks. Since $H(t)$ is uncertain it might influence the control proposition. From now on, we will consider $H(t)$ uncertain. If Assumption 1 is verified, we can used TDE [11] to estimate $H(t)$ as follows:

$$
\widehat{H}(t) \approx H\left(t-t_{d}\right)=U\left(t-t_{d}\right)-f\left(t-t_{d}\right)-\dot{z}_{2}\left(t-t_{d}\right)
$$

where, $t_{d}$ is a very-small time delay constant. Practically, the smallest constant that can be achieved in real time is the sampling period. According to the Lipschitz condition (Assumption 1), the time delay error can be calculated as follows:

$$
\Delta H=H(t)-\widehat{H}(t)=H(t)-H\left(t-t_{d}\right) \leq \varrho t_{d}
$$

where $\varrho>0$ is the Lipschitz constant.

Remark 2: It can be seen from Eq.16 that if Assumption 2 is verified, the estimation error of the uncertainties and disturbances is always bounded by the Lipschitz constant.

Theorem 2: Considering the exoskeleton robot system (Eq. 3) which satisfies the mentioned properties and assumptions, the control law of Integral Second-Order Terminal Sliding Mode Control incorporating TDE ensures the convergence of the sliding surface and its first and second derivative to zero in finite-time given by:

$$
U(t)=-K_{1}|\dot{S}|^{\frac{1}{2}} \operatorname{sign}(S)+\ddot{Z}^{d}-f(t)-\widehat{H}(t)+\sum_{i=1}^{7} \frac{\beta \lambda_{2 i}{ }^{2}}{\lambda_{1 i}{ }^{2}}|e|^{2 \beta-1} \operatorname{sign}\left(e_{i}\right)-\int_{0}^{t} K_{2} \operatorname{sign}(S)
$$

where $K_{1}=k_{1} \lambda_{1}=\operatorname{diag}\left(K_{1 i i}\right)>0$ and $K_{2}=k_{2} \lambda_{1}=\operatorname{diag}\left(K_{1 i i}\right)>0$, where $i=1, \ldots, 7$. Whenever the following conditions are verified:

$$
K_{1 i}>2 \varrho t_{d}, K_{2 i}>\frac{\varrho t_{d}\left(K_{1 i}\right)^{2}-K_{1 i}{ }^{3}}{2\left(3 K_{1 i}-2 \varrho t_{d} K_{1 i}\right)}
$$

Proof: Before selecting the Lyapunov function candidate, let us substitute the control law (Eq. 17) into Eq.13, we find:

$$
\left\{\begin{array}{l}
\ddot{S}=-K_{1}|\dot{S}|^{\frac{1}{2}} \operatorname{sign}(S)+\lambda_{1} \Delta H+w \\
\dot{w}=-K_{2} \operatorname{sign}(S)
\end{array}\right.
$$

It can be seen that Eq. 19 has the same structure as the Super-Twisting control [8]. Let us now introduce new variables such that: $\eta_{1}=S$ and $\eta_{2}=\dot{S}$. The system (Eq. 19) becomes as follows:

$$
\left\{\begin{array}{l}
\dot{\eta}_{1}=\eta_{2} \\
\dot{\eta}_{2}=-K_{1}\left|\eta_{2}\right|^{\frac{1}{2}} \operatorname{sign}\left(\eta_{1}\right)+\lambda_{1} \Delta H+w \\
\dot{w}=-K_{2} \operatorname{sign}\left(\eta_{1}\right)
\end{array}\right.
$$

To ensure the convergence of the robot system (Eq. 3), we will assume the following Lyapunov function candidate:

$$
V=\gamma^{T} R \gamma
$$


where $\gamma=\left[\gamma_{1 i}, \gamma_{2 i}\right]^{T}, \gamma_{1 i}=\left(\left|\eta_{2 i}\right|\right)^{\frac{1}{2}} \operatorname{sign}\left(\eta_{1}\right), \gamma_{2 i}=w_{i}$. The Lyapunov function (Eq. 21) is chosen to be continuous and non-differentiable at $S_{i}=0$ [16]. It is positive-definite and radially-bounded by choosing an appropriate matrix $R \in \mathbb{R}^{2 \times 2}$ such that,

$$
R=\frac{1}{2}\left[\begin{array}{cc}
K_{1 i}^{2}+4 K_{2 i} & -K_{1 i} \\
-K_{1 i} & 2
\end{array}\right]
$$

with,

$$
\alpha_{\min }\{R\}\|\gamma\|^{2} \leq V \leq \alpha_{\max }\{R\}\|\gamma\|^{2}
$$

where $\alpha_{\min }\{R\}$ and $\alpha_{\max }\{R\}$ are the minimum and maximum eigenvalues of $\{R\}$ and $\|\gamma\|$ is the Euclidian norm of $\gamma$. Taking the derivative of Lyapunov function (Eq. 21):

$$
\dot{V}=\dot{\gamma}^{T} R \gamma+\gamma^{T} R \dot{\gamma}
$$

The time derivative of $\gamma$ can be defined as follows:

$$
\left\{\begin{array}{l}
\dot{\gamma}_{1 i}=\frac{1}{2\left|\eta_{2 i}\right|^{\frac{1}{2}}} \dot{\eta}_{2 i} \\
\dot{\gamma}_{2 i}=\dot{w}_{i} ; i=1, \ldots, 7
\end{array}\right.
$$

Using Eq.20 and Eq. 24, we can rewrite $\dot{\gamma}$ in matrix form, where $\left|\gamma_{1 i}\right| \leq\left|\eta_{2 i}\right|^{\frac{1}{2}}$ :

$$
\dot{\gamma}=\frac{1}{\left|\gamma_{1 i}\right|}\left[\begin{array}{cc}
\frac{-K_{1 i}}{2} & \frac{1}{2} \\
-K_{2 i} & 0
\end{array}\right]\left[\begin{array}{l}
\gamma_{1 i} \\
\gamma_{2 i}
\end{array}\right]+\frac{1}{\left|\gamma_{1 i}\right|}\left[\begin{array}{l}
\frac{\lambda_{1}}{2} \\
0
\end{array}\right] \Delta H_{i}
$$

The above equation can be written in the form:

$$
\dot{\gamma}=\frac{1}{\left|\gamma_{1 i}\right|}\left(A_{s} \gamma+B_{S} \Delta H_{i}\right)
$$

where, $A_{S}=\left[\begin{array}{cc}\frac{-K_{1 i}}{2} & \frac{1}{2} \\ -K_{2 i} & 0\end{array}\right] ; B_{S}=\left[\begin{array}{c}\frac{\lambda_{1}}{2} \\ 0\end{array}\right]$. Substituting Eq. 26 into Eq. 23, we find:

$$
\dot{V}=\frac{1}{\left|\gamma_{1 i}\right|} \gamma^{T}\left(A_{S}^{T} R+R A_{S}\right) \gamma+\frac{2}{\left|\gamma_{1 i}\right|} \Delta H_{i} B_{S}^{T} R \gamma
$$

Since $\varrho t_{d}$ is positive from Eq. 16. The following inequality can be established: $2 \Delta H_{i} B_{s}^{T} R \gamma \leq \varrho t_{d} \gamma^{T} M \gamma$, where:

$$
M=\frac{1}{2}\left[\begin{array}{cc}
K_{1 i}^{2}+4 K_{2 i} & \frac{-1}{2} K_{1 i} \\
\frac{-1}{2} K_{1 i} & 0
\end{array}\right]
$$

Therefore Eq.27 becomes as:

$$
\dot{V} \leq \frac{1}{\left|\gamma_{1 i}\right|} \gamma^{T}\left(A_{s}^{T} R+R A_{s}+\varrho t_{d} M\right) \gamma
$$


The above Eq. 28 can be rewrite as follows:

$$
\dot{V} \leq \frac{1}{\left|\gamma_{1 i}\right|} \gamma^{T} D \gamma
$$

where $D$ is written such that $D=-\left(A_{s}^{T} R+R A_{s}+\varrho t_{d} M\right)$, and $D$ is calculated such that:

$$
D=\frac{-K_{1 i}}{2}\left[\begin{array}{cc}
K_{1 i}^{2}+6 K_{2 i}-\varrho t_{d}\left(K_{1 i}+4 \frac{K_{2 i}}{K_{1 i}}\right) & \frac{1}{2} \varrho t_{d}-K_{1 i} \\
\frac{1}{2} \varrho t_{d}-K_{1 i} & 1
\end{array}\right]
$$

The function $\dot{V}$ is negative definite if $K_{1 i}>2 \varrho t_{d}, K_{2 i}>\frac{\varrho t_{d}\left(K_{1 i}\right)^{2}-K_{1 i}{ }^{3}}{2\left(3 K_{1 i}-2 \varrho t_{d} K_{1 i}\right)}$. This selection will ensure that $\operatorname{the} \operatorname{det}(D)>0$. While $D$ is positive and symmetric. In such case, we can rewrite Eq. 29 as:

$$
\dot{V} \leq \frac{-1}{\left|\gamma_{1 i}\right|} \alpha_{\min }\{D\}\|\gamma\|^{2}
$$

where $\alpha_{\min }\{D\}$ is the minimum eigenvalue of $D$. Eq. 31 proves that Lyapunov function is semi-negative definite. Now, let us prove the finite time convergence of the system. From Eq. 22, we have:

$$
\frac{V^{\frac{1}{2}}}{\alpha_{\max }^{\frac{1}{2}}\{R\}} \leq\|\gamma\|^{2} \leq \frac{V^{\frac{1}{2}}}{\alpha_{\text {min }}^{\frac{1}{2}}\{R\}}
$$

It is clear that: $\left|\gamma_{1 i}\right| \leq\|\gamma\|$ and from Eq. 31 and Eq. 32, we have:

$$
\dot{V} \leq \frac{-1}{\left|\gamma_{1 i}\right|} \alpha_{\min }\{D\}\|\gamma\|^{2} \leq \frac{\alpha_{\min }\{D\}}{\alpha_{\max }^{\frac{1}{2}}\{R\}}
$$

According to this equation, the finite time convergence of the sliding surface can be obtained such that:

$$
T_{S}=\frac{2 \alpha_{\max }^{\frac{1}{2}}\{R\}}{\alpha_{\min }\{D\}} V^{\frac{1}{2}}(\gamma(0))
$$

\section{Experiment and Comparative Study}

The robot system consists of three processing units, the first is a PC where the top-level commands are sent to the robot using a LabVIEW interface, i.e. the control scheme selection. This PC also receives the data after the robot task is executed to analyze its performance. The other two processing units are part of a National Instruments PXI platform. Firstly, a NI-PXI 8081 controller card with an Intel Core Duo processor; in this card, the main operating system of the robot and the top-level control scheme are executed. In our case, the ISOTSMC based controller as well as the estimation based on time delay approach, at a sampling time of 500 $\mu$ s. Finally, at input/output level, a NI PXI-7813R remote input/output card with a FPGA (field programmable gate array) executes the low-level control; i.e. a PI current control loop (sampling time of $50 \mu \mathrm{s}$ ) to maintain the current of the motors required by the main controller. Also, in this FPGA, the position feedback via Hall-sensors (joint position) and basic input/output tasks are executed. Each joint of the ETSMARSE is powered by a brushless DC motor (Maxon EC-45, EC-90 ) combined with harmonic drives (gear ratio 120:1 for motor-1, motor-2, and motor-4 and gear ratio 100:1 for motor-3 and motors 5-7). 

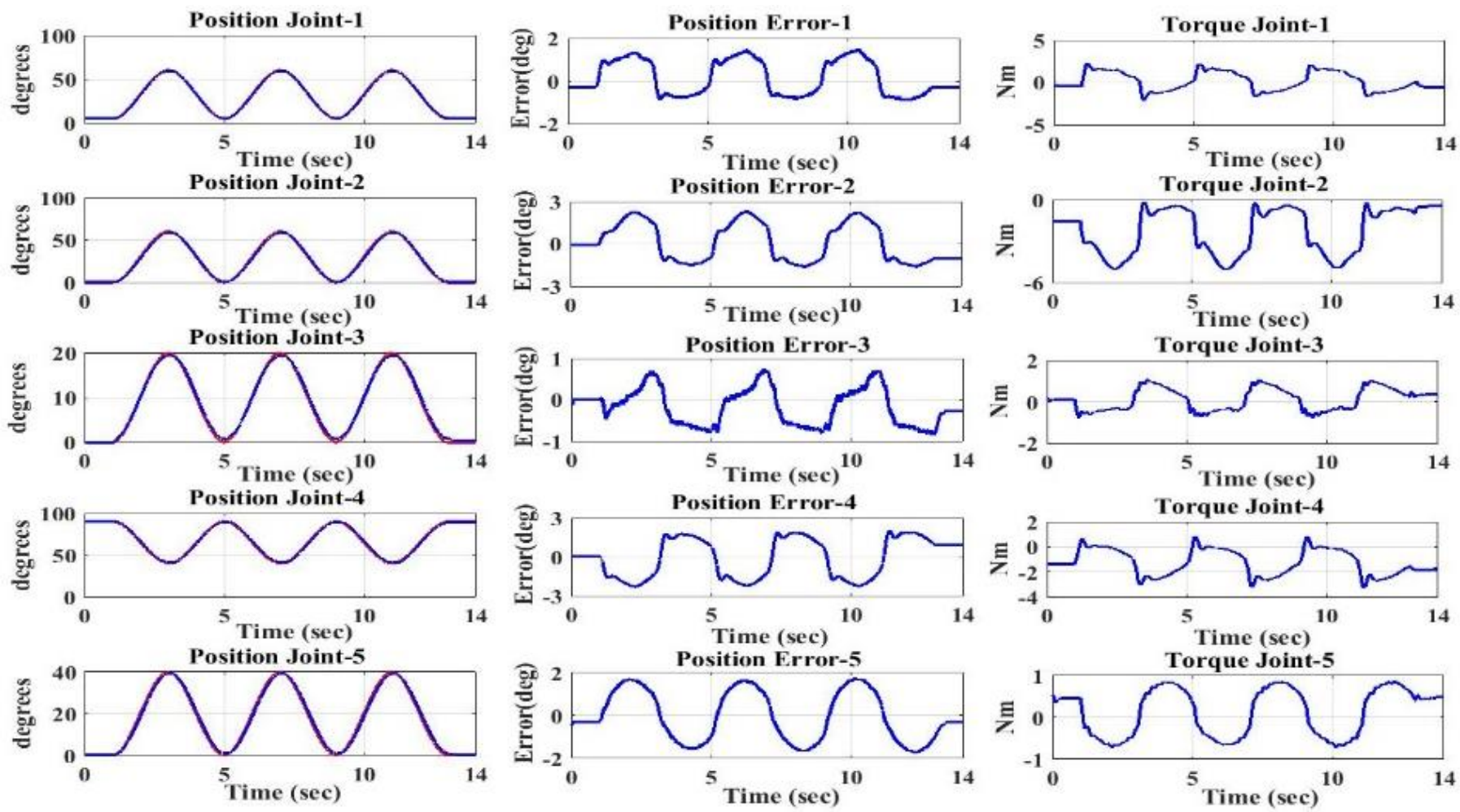

Fig. 2: Performance of the ETS-MARSE robot with subject-A in joint space.

An experiment session was created to validate the proposed control approach. It consists on an exercise performed with a healthy subject with an age of 30 years, height of $177 \mathrm{~cm}$, and weight of $75 \mathrm{Kg}$. In this case, the trajectory is repeated three times for each movement with the speed varying between $(28 \mathrm{deg} / \mathrm{sec}$ for joint-3 and $48 \mathrm{deg} / \mathrm{sec}$ for the remaining joints). The results of the task are illustrated in Fig. 4. The initial position of the robot is given with the elbow joint position at 90 degrees. The control gains are chosen manually as follows: $\lambda_{1 i}=2.5, \lambda_{2 i}=12.5 ; k_{1}=18, k_{2}=10$, $\beta=0.6$.

We can appreciate in this figure that for the movement of all joints, the desired trajectory (represented by the red line), practically overlaps the measured trajectory (represented by the solid blue line). It is clear from the plots in this figure that the proposed controller provides an excellent performance. Where, the controller has the potential to maintain stability of the system along the designed therapeutic movement with a position error (second column of Fig. 2) less than three degrees for all joints. The last column of Fig. 2 shows the control input which is clearly smooth and without the chattering effect. We can conclude that the controller is robust; it offers a very good performance despite the high speed and unknown parameters of the robot.

The proposed ISOTSMC controller is compared with conventional second-order sliding mode controller SOSMC to show the feasibility and advantage of the proposed controller. The comparison is made in terms of tracking position error, and torque input by computing the Root-Mean Square (RMS)

Table 1: Controllers evolution.

\begin{tabular}{|c|c|c|}
\hline Controller & $\begin{array}{l}\text { RMS } \\
\text { (error) }\end{array}$ & RMS (Torque) \\
\hline ISOTSMC & 0.0150 & 2.0728 \\
\hline SOSMC & 0.0988 & 3.2147 \\
\hline
\end{tabular}


It is clear from Table I that the proposed controller achieves an excellent performance with small value of overall RMS error, even when the dynamic model of the exoskeleton is not completely known, and in presence of external forces.

\section{Conclusion}

In this paper, we investigated the control applied to passive rehabilitation protocol of an exoskeleton robot by presenting a new integral second-order terminal sliding mode incorporating time delay estimation. Using second-order sliding mode is due to its attractive characteristics of fast convergence, accuracy, and attenuation of chattering. However, its problem is that the unknown dynamic of the exoskeleton robot and external disturbances can be amplified by the second derivative of the sliding surface, which leads to instability of the robot system. Applying TDE to estimate the unknown dynamics and external disturbances permits chattering reduction. The controller is dedicated to improve the robustness of the second-order sliding mode control while overcoming its main limitation. The stability analysis is formulated and demonstrated based on Lyapunov function. An experimental physiotherapy session with a healthy subject was created to test the effectiveness and feasibility of the proposed control, which are proved.

\section{Acknowledgements}

This research was supported by The Power Electrics and Industrial Control research Group (GRÉPCI), École de Technologie Supérieure, Montreal, Canada.

\section{References}

[1] S. Xie, "Advanced Robotics for Medical Rehabilitation," Springer Tracts in Advanced Robotics, vol. 108, pp. 1-357, 2016.

[2] J.-J. E. Slotine and W. Li, Applied nonlinear control, vol. 199. Prentice hall Englewood Cliffs, NJ, 1991.

[3] Y. Wu, X. Yu, and Z. Man, "Terminal sliding mode control design for uncertain dynamic systems," Systems \& Control Letters, vol. 34, pp. 281-287, 1998.

[4] X. Yu and M. Zhihong, "Fast terminal sliding-mode control design for nonlinear dynamical systems," IEEE Transactions on Circuits and Systems I: Fundamental Theory and Applications, vol. 49, pp. 261-264, 2002.

[5] Y. Feng, X. Yu, and Z. Man, "Non-singular terminal sliding mode control of rigid manipulators," Automatica, vol. 38, pp. 2159-2167, 2002.

[6] L. M. Fridman, "An averaging approach to chattering," IEEE Transactions on Automatic Control, vol. 46, pp. 1260$1265,2001$.

[7] A. Levant, "Principles of 2-sliding mode design," Automatica, vol. 43, pp. 576-586, 2007.

[8] T. Gonzalez, J. A. Moreno, and L. Fridman, "Variable gain super-twisting sliding mode control," IEEE Transactions on Automatic Control, vol. 57, pp. 2100-2105, 2012.

[9] S. Ding, J. Wang, and W. X. Zheng, "Second-order sliding mode control for nonlinear uncertain systems bounded by positive functions," IEEE Transactions on Industrial Electronics, vol. 62, pp. 5899-5909, 2015.

[10] L. Zhao, J. Huang, H. Liu, B. Li, and W. Kong, "Second-order sliding-mode observer with online parameter identification for sensorless induction motor drives," IEEE Transactions on Industrial Electronics, vol. 61, pp. 5280$5289,2014$.

[11] B. Brahmi, M. Saad, C. Ochoa-Luna, and M. H. Rahman, "Adaptive control of an exoskeleton robot with uncertainties on kinematics and dynamics," in 2017 International Conference on Rehabilitation Robotics (ICORR), , 2017, pp. 1369-1374.

[12] B. Brahmi, M. Saad, C. O. Luna, P. Archambault, and M. Rahman, "Sliding mode control of an exoskeleton robot based on time delay estimation," in 2017 International Conference on Virtual Rehabilitation (ICVR), 2017, pp. 1-2.

[13] A. M. Shotorbani, A. Ajami, S. G. Zadeh, M. P. Aghababa, and B. Mahboubi, "Robust terminal sliding mode power flow controller using unified power flow controller with adaptive observer and local measurement," IET Generation, Transmission \& Distribution, vol. 8, pp. 1712-1723, 2014.

[14] M. H. Rahman, M. J. Rahman, O. Cristobal, M. Saad, J.-P. Kenné, and P. S. Archambault, "Development of a whole arm wearable robotic exoskeleton for rehabilitation and to assist upper limb movements," Robotica, vol. 33, pp. 1939, 2015. 
[15] H. Wang, Z.-z. Han, Q.-y. Xie, and W. Zhang, "Finite-time chaos synchronization of unified chaotic system with uncertain parameters," Communications in Nonlinear Science and Numerical Simulation, vol. 14, pp. 2239-2247, 2009.

[16] J. A. Moreno and M. Osorio, "A Lyapunov approach to second-order sliding mode controllers and observers," in 47th IEEE Conference on Decision and Control, 2008. CDC 2008, pp. 2856-2861, 2008. 